\title{
The State of the Atomic Lattice of Structure Materials: Pre NDE Indication Assessments
}

\author{
F. M. Al-Abbas ${ }^{1, *}$, F. A. Al-Hindas ${ }^{1}$, D. L. Olson ${ }^{2}$, B. Mishra ${ }^{2}$ \\ ${ }^{1}$ Department of Inspection, Saudi Aramco, Saudi Arabia \\ ${ }^{2}$ Department of Metallurgical and Materials Engineering, Colorado School of Mines, USA
}

Copyright $(\subset 2015$ by authors, all rights reserved. Authors agree that this article remains permanently open access under the terms of the Creative Commons Attribution License 4.0 International License

\begin{abstract}
The lattice of structural alloys possesses electronic characteristics which offer NDE predictions of potential failures in materials and structural assemblies. The atomic lattice represents a periodic potential which provide electronic wave descriptions that appear to be free. Any distortion in the periodic lattice (potentials) will achieve electronic and / or elastic interactions giving advance NDE waves tools possibility to assess stability of engineering materials at the atomic level during service life. Furthermore, the small dimensional defects (i.e. dislocations and interfaces) will produce steep potential gradients resulting in electronic and/or elastic physical properties changes that can be also measured. These collective potential and electronics signatures could be used as pre NDE lattice indications to produce awareness of potential degradations (i.e. susceptibility of fatigue, strain enhanced corrosion and cracking). The proper selection, utilization and assessment will increase both reliability and the NDE economics. A new approach to the next generation of nondestructive evaluations based on the electronic and elastic wave signals and analyses will be presented.
\end{abstract}

Keywords NDE, Indication, Structure, Lattice, Material

\section{Introduction}

Processed materials consist of compositional,

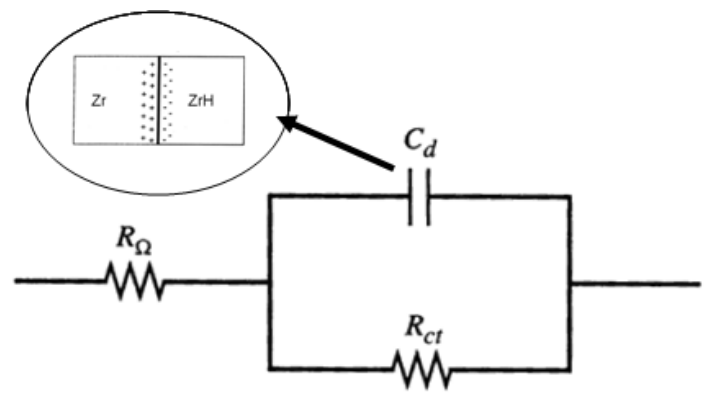

Figure 1. (Left) A model circuit of the material sensed by low frequency impedance, comprised of capacitive interfaces between resistive grains. (Right) A proposed example of the charge distribution in fine grained zircalloy with hydrides present.

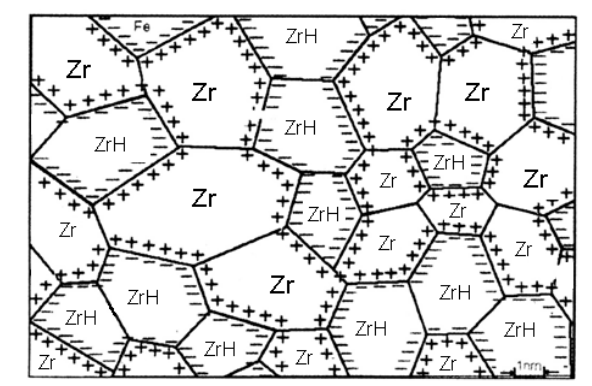

microstructural, and property gradients which require modifications in the materials science fundamentals to describe the non-uniform materials behavior, such as aging, phase stability, and defect initiation, especially in materials that depend on nano-structural features. When lattice dimensions in the micro or nano range are necessary to describe the material [1], then the nonlinear thermodynamics and kinetics are used to accurately describe the system. Tu [1] expressions describing the transport and phase evolution of nano-scale thin films.

Advanced composite theory will need to describe the electronic charge gradient across the bonding interfaces based on electronic concepts. Fig. 1 illustrates an electronic junction resulting from a metal-metal hydride interface which will behave electronically as a local capacitor to an eddy current. These bonding interfaces will have steep electronic gradients in a metal described by the very short Debye length. The expressions describing such steep potentials will require additional nonlinear terms. The influence of these nonlinear effects on NDE assessment needs to be investigated and the analytical materials science practices need to be developed. These practices will allow interpretation of the micro or nano structural state of many advanced and high performance materials. Inclusions in the metallic matrix also have an electronic junction at their interfaces and this junction can be treated also as a charged dipole [2-5]. described the need for application of nonlinear terms in the 


\section{Nonlinear Behavior of Defects in Solids}

The nonlinear interpretation can be extended to electron charge distribution of a defect in a crystal, such as the charge distribution of a dislocation in a metal. The dislocation is represented as an extra half-plane of atoms above the slip plane and can also be described as an electric dipole along the dislocation line as shown in Fig. 2.

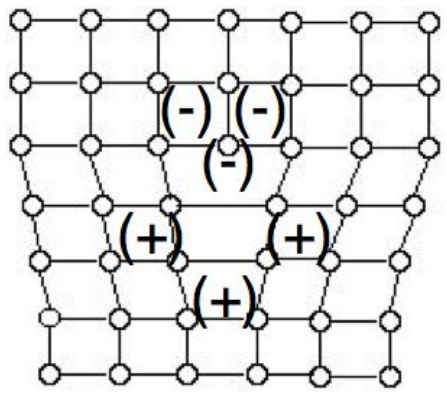

Figure 2. Electronic description of an edge dislocation as an electronic dipole

Applying the Poisson equation to this charge distribution will allow the dislocations to be described as steep electric potential gradients [3], such as:

$$
\phi=\phi_{0} e^{-\frac{|x|}{\lambda_{0}}}
$$

The potential of this dislocation in a metal can be experienced only in extremely short distances due to the very small Debye length for a metal which is given as:

$$
\lambda_{0}=\sqrt{\frac{\varepsilon_{0} k T}{e^{2} n_{\infty}}}
$$

where $n_{\infty}$ is the electron concentration for a metal. The interaction of a dislocation with an inclusion can be considered a dipole-dipole interaction which is a very short-range interaction. Since these potential gradients are very steep in a metal with its large electron concentration, the electrical potential obtained by the Poisson equation should be nonlinear. This nonlinear electrical potential can be assessed by measuring the higher order electric properties terms, such as through harmonic analysis of the induced voltage. This practice will offer new insight into dislocation behavior by measuring the higher harmonic frequencies.

Zander et al. [4] have reported that hydrogen-charged 2.25 $\mathrm{Cr}-1 \mathrm{Mo}$ steel deforms as if it is pure iron. This behavior is illustrated in Fig. 3. They suggest that hydrogen allows the dislocation to not be hindered or pinned by the inclusions as shown by the change in compressive strength in $2.25 \mathrm{Cr}-1 \mathrm{Mo}$ steel with or without the addition of hydrogen. An explanation of this mechanical behavior as electronic hydrogen interaction can be described as a dipole-dipole interaction as shown in Fig. 4. Solute hydrogen in some transition metals such as iron has been reported as giving its electron to the d-band and moves in the lattice as a proton. This concept is also suggested in Fig. 4.

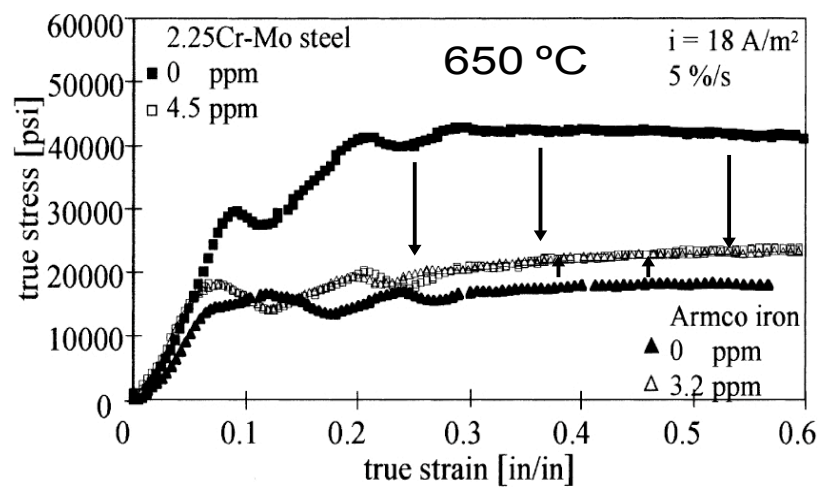

Figure 3. The influence of hydrogen content in $2.25 \mathrm{Cr}-1 \mathrm{Mo}$ steel on its compressive strength. The comparison of this inclusion-strengthened steel with pure iron illustrates that with sufficient hydrogen content, $2.25 \mathrm{Cr}-1 \mathrm{Mo}$ steel behaves mechanically as pure iron [4].

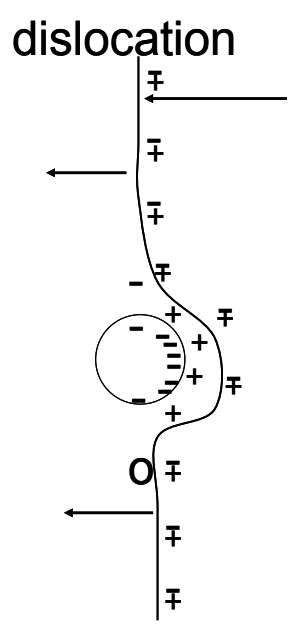

(a)

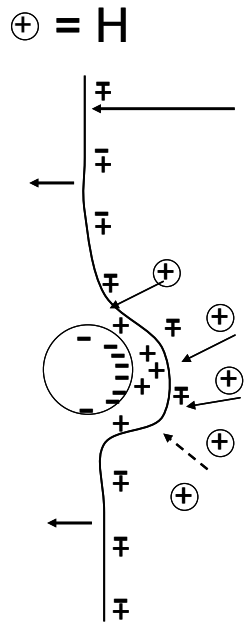

(b)
Figure 4. (a) Dipole-dipole interaction between a dislocation and inclusion (b) Illustration of soluble hydrogen (proton) altering the dipole-dipole interaction between the dislocation and the inclusion altering the mechanical behavior [3,4].

\section{The Role Electron in Solid State}

The commonality of advanced NDE techniques occurs at the electronic level. All of the NDE techniques assess the electronic structure of materials and perturbations in the structure due to defects, microstructural phases and their features, manufacturing and processing, and service-induced strains. Electronic, magnetic, and elastic properties have all been correlated to fundamental electronic properties of the material and thus to structural imperfections. The commonality between elastic and electronic impedances and their resonance frequencies for coupling applied force oscillations is given in Table 1. 
Table 1. Similarity of elastic and electronic impedances [3]

\begin{tabular}{|c|c|}
\hline Mass on a Spring & $\begin{array}{c}\text { Circuit containing Inductance } \\
\text { and Capacitance }\end{array}$ \\
\hline$\frac{1}{2} m v^{2}+\frac{1}{2} k x^{2}=\frac{1}{2} k A^{2}$ & $\frac{1}{2} L i^{2}+\frac{1}{2} \frac{q^{2}}{C}=\frac{1}{2} \frac{Q^{2}}{C}$ \\
\hline$Z_{m}=\left(R^{2}+\left(\omega m-\frac{k}{\omega}\right)^{2}\right)^{\frac{1}{2}}$ & $Z_{e}=\left(R^{2}+\left(\omega L-\frac{1}{\omega C}\right)^{2}\right)^{\frac{1}{2}}$ \\
\hline$\omega=2 \pi f=\sqrt{\frac{k}{m}}$ & $\omega=2 \pi f=\sqrt{\frac{1}{L C}}$ \\
\hline
\end{tabular}

Electronic band theory, Fermi energy, and Brillouin zones are all based on wave mechanics concepts [3]. The use of the free electron model for a metal can be extended to consider electron-lattice potential interactions through the introduction of the effective mass of the electron, $m_{e}[3,5]$. The total energy (E) of an electron in a solid, based on wave mechanics is:

$$
E=\frac{\mathrm{h}^{2} k^{2}}{2 m}+V
$$

where $k$ is the electronic wave vector, and $V$ is the potential that the nearly free electron is experiencing from the lattice. The information of the potential of electron-lattice interactions $(V)$, is incorporated into the electron mass factor $m$, then the total energy of electron in the lattice can be expressed in terms of effective mass $m_{e}$ as:

$$
E=\frac{h^{2} k^{2}}{2 m_{e}}
$$

These localized potentials represent the structural contributions that disturb the periodic lattice (Bloch function), such as dislocations, grain boundaries, areas of lattice strain, and phase changes. The effective mass of the electron, $m_{e}$, is very sensitive to lattice changes in alloy and is derived to be:

$$
m_{e}=\frac{h^{2}}{\left(\frac{d^{2} E}{d k^{2}}\right)}
$$

where $\hbar$ is the Planck constant divided by $2 \pi$. Electronic property measurements offer a very sensitive NDE assessment tool of microstructural constituents and alloy stability, based on the second derivative factor in Equation (5).

For example, when the Fermi energy surface and alloying contacts the Brillouin zone boundary, which represents an energy band gap that exists when the electron energy has a wave vector that will be diffracted, the conditions for a phase transformation occurs. After this it quickly fills higher electronic energy states during further alloy additions, thus rapidly increasing the effective mass of the electron. To minimize energy, the lattice will select a different crystal structure, thus a new Brillouin zone, allowing for lower energy filling. This situation is the electronic explanation for phase transformations. If it is possible to calculate, such as the use of Phacomp to predict whether a specific alloy composition is susceptible for sigma phase formation in superalloys [5], then it should be possible to measure the electronic property state for sigma phase formation.

\section{NDE Measurements for Materials Characterization}

Dobmann et al. [6] correlated the dimensions of microstructural defects to various NDE assessment technologies as shown in Fig. 5. They illustrated that understanding the physical limitation of each NDE assessment practice is essential for characterization for complex high performance alloys.

The complex aging kinetics in alloys offers a challenging opportunity to examine the concept of fully characterizing a material using multiple NDE tools. The aging kinetics of maraging steels involves precipitation of metastable phases $\left(\mathrm{Ni}_{3}(\mathrm{Ti}, \mathrm{Mo})\right)$ followed by the formation of stable phases $\left(\mathrm{Fe}_{2}(\mathrm{Mo}, \mathrm{Ti})\right)$ and austenite with continued compositional changes in the highly alloyed matrix. Also, high residual strains caused by the semi-coherent precipitates and reduction in the dislocation and point defect densities have offered a number of indications for different alloy materials. These indications could be used to fully characterize a material using multiple nondestructive evaluations. Raj, Rajkumar et al. [7-10] compared various nondestructive and destructive testing techniques on heat treated Maraging 250 steel. It is apparent that these NDE techniques with proper selection can assess the aging as well as the traditional hardness technique. Some techniques show that the aging involves a series of precipitation events. 


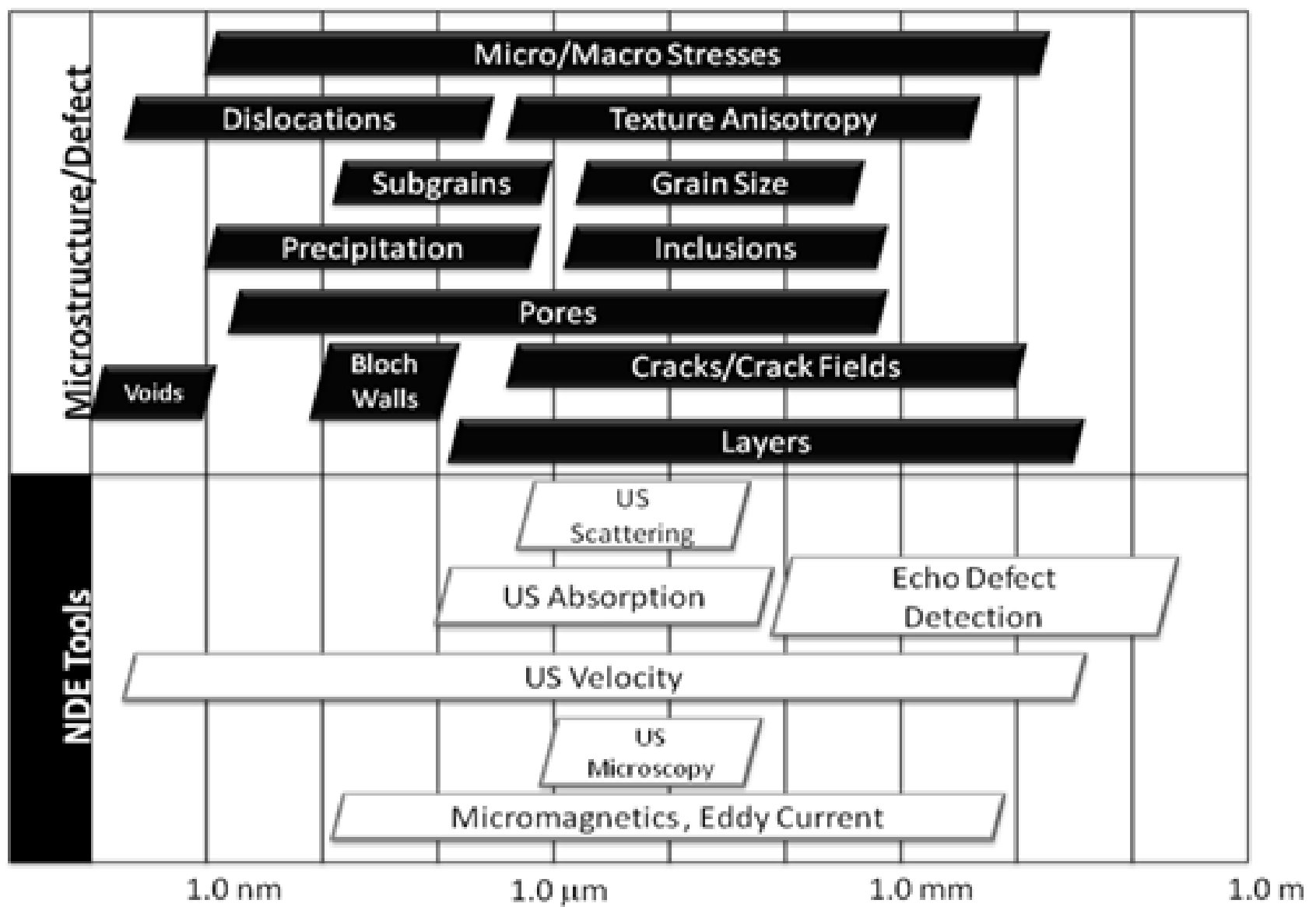

Figure 5. Correlation of dimensionality of lattice defects to the effective range of the various NDE analytical assessment tools (adopted from [6]).

\section{The Need for Multiple Measurements}

The significant difficulty of using a single physical measurement to characterize material microstructure, composition, or other properties is that they are dependent on numerous independent variables. Electronic property measurements are dependent on at least three independent electronic properties. For a physical property measurement to assess the material state requires reference to material standards, or correlations with multiple independent measurements, such as use of both elastic and electronic wave measurements or use wave measurements made with different frequencies, and wavelengths.

The necessary advancement in use of physical measurements to assess materials is through the use and correlation of sufficient different physical measurements to experience all the independent material variables. The use of property measurements, based on wave analysis (electromagnetic and elastic waves), allows measurements to be made at different frequencies that interact with different specific microstructural details. The use of the same physical measurements at different frequencies can lead to correlation of material independent variables. By implementing a full range of wave perturbing frequencies and with knowledge of the depth factor, wavelengths can be used at all levels of microstructural scale, from nanometer to millimeter, to assess atomic to grain structure, size and morphology. The interplay between wave perturbations and the matter (and the reverse) will become an even more valuable assessment protocol. Both frequency and amplitude modulation can be applied to this wave analysis. With instrumentation, various combinations of harmonics between the perturbation wave and the analyzed wave may be utilized to assist in signal recognition analysis. The use of wave analysis, whether electronic, magnetic, or elastic, offers the new capabilities to assess the material without using a calibration standard since specific wavelength dimension correlates with specific microstructural constituents. 

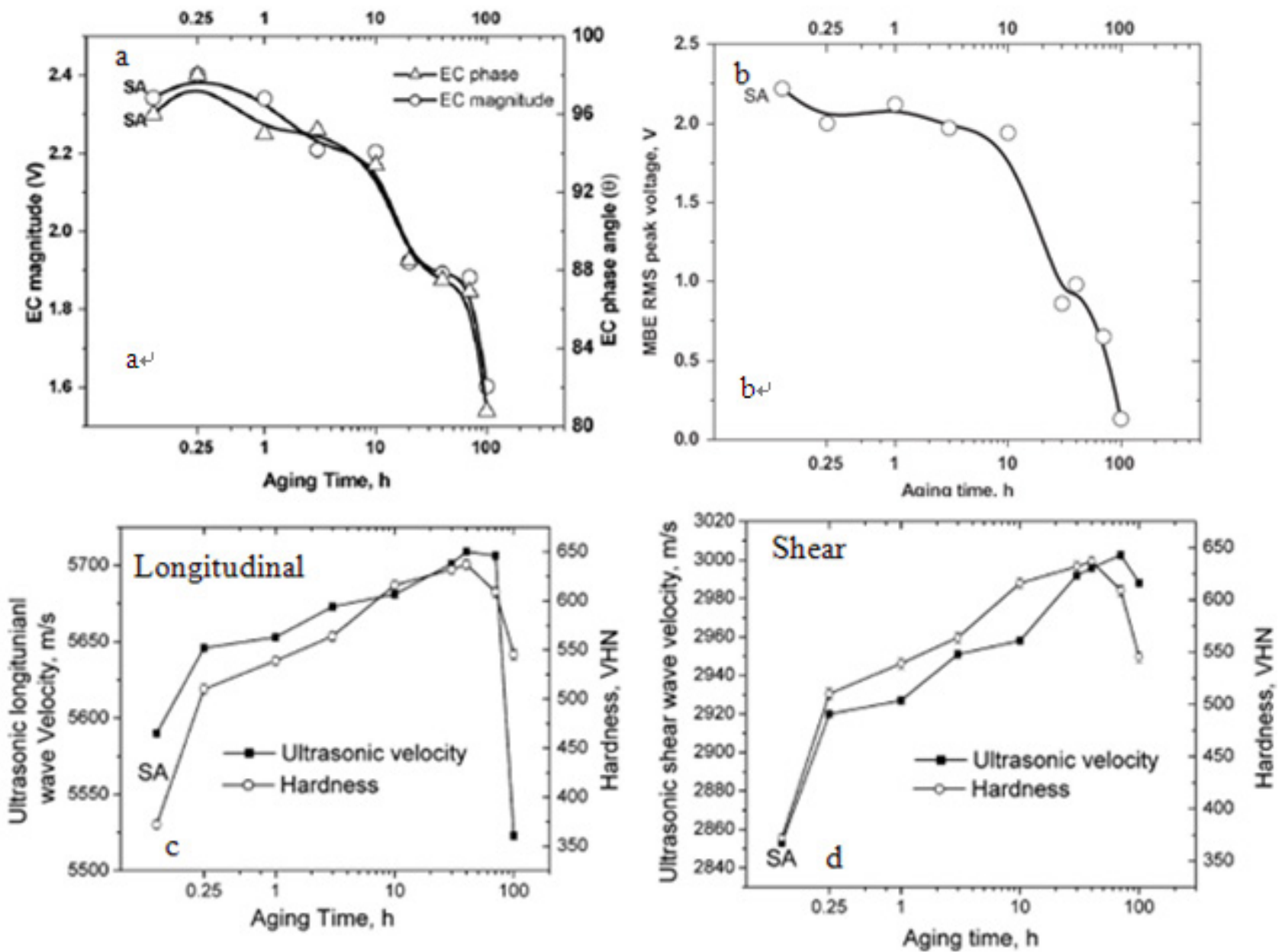

Figure 6. The variation in physical property measurements (Eddy current, Magnetic, Ultrasonic) of Maraging 250 alloy as a function of aging time at $755 \mathrm{~K}$ : (a) Eddy current, (b) Magnetic Barkhausen Noise, and (c) Ultrasonic Longitudinal Wave Velocity, (d) Ultrasonic Shear Wave Velocity [7-10].

\section{The Need for a Microstructural Rule}

As with material phase analysis, the phase rule determines the least number of intrinsic properties, the degrees of freedom (F), to define the number of phases. A microstructural rule is needed to determine the specific number of intrinsic property measurements to fully characterize a microstructure [11]. A suggested Microstructure Rule is given as:

$$
F=C-P+2+M
$$

where $C$ is the number of components and $P$ is the number of phases, the "2" recognizes pressure and temperature. The additional $M$ term addresses the need for additional instrinic measurements to express microstructural constituents' concentrations, sizes, and morphologies. Consider the example of the two phases, $\alpha+\mathrm{Fe}_{3} \mathrm{C}$, region of the iron-carbon system. This region can be represented by various morphologies such as pearlite, bainite, or as spherical carbides in a ferrite matrix. All three satisfy the same phase rule count of phases, but additional property measurements are needed to distinguish the specific microstructural features. The effort to establish a quantitative scheme to determine $M$ will have to draw rules from phase equilibrium, phase transformation, metallography, petrology, and topology.

\section{Magnetic Wave Measurements}

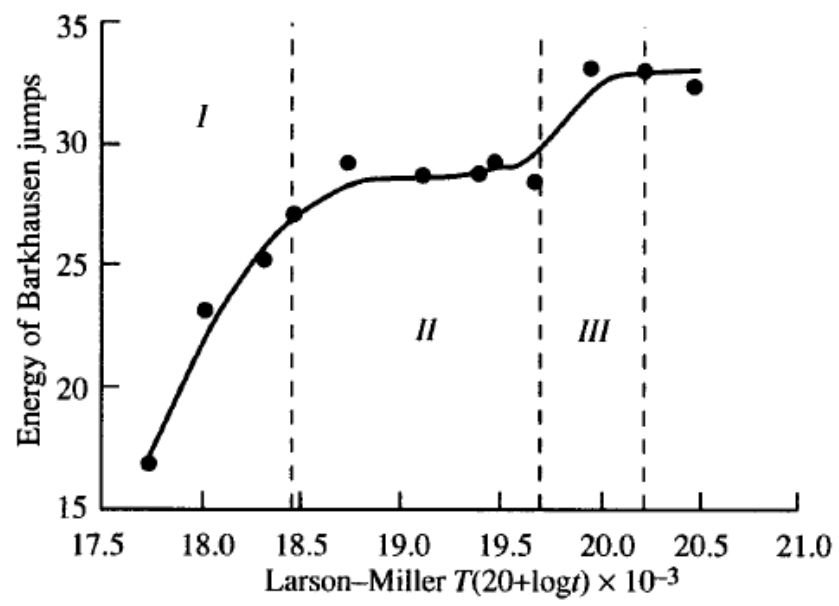

Figure 7. Energy of Barkhausen jumps per one magnetization cycle as a function of the Larson-Miller parameter on the three stages of microstructure evolution [13]. 
Magnetic Barkhausen and Magnetic Acoustic Emissions analyses assess the elastic emission resulting from the transporting of the magnetic domain wall through ferromagnetic materials [12]. The elastic emission results from the unpinning events of the Bloch wall from non-periodic sites in the lattice. Fig. 7 shows the integration of the Barkhausen signal which represents the energy of Barkhausen jumps per magnetization cycle. This energy can be correlated to the microstructural evolution of $12 \mathrm{CrMoV}$ steel during isothermal annealing.

\section{Electronic Wave Measurements}

The use of electronic impedance (eddy current) measurements have been used to assess residual stress [14] and strain associated with the machining [15], hydrogen content in steel pipe [16-18], even thru organic coating [19], and in Zircaloy 4, under water [20]. With proper calibration and standardization a low frequency eddy current practice can be used as convenient and accurate nondestructive and non-contact method to measure the hydrogen in structural alloys. Fig. 8 illustrates the ability to measure hydrogen content in linepipe steel using low frequency impedance.

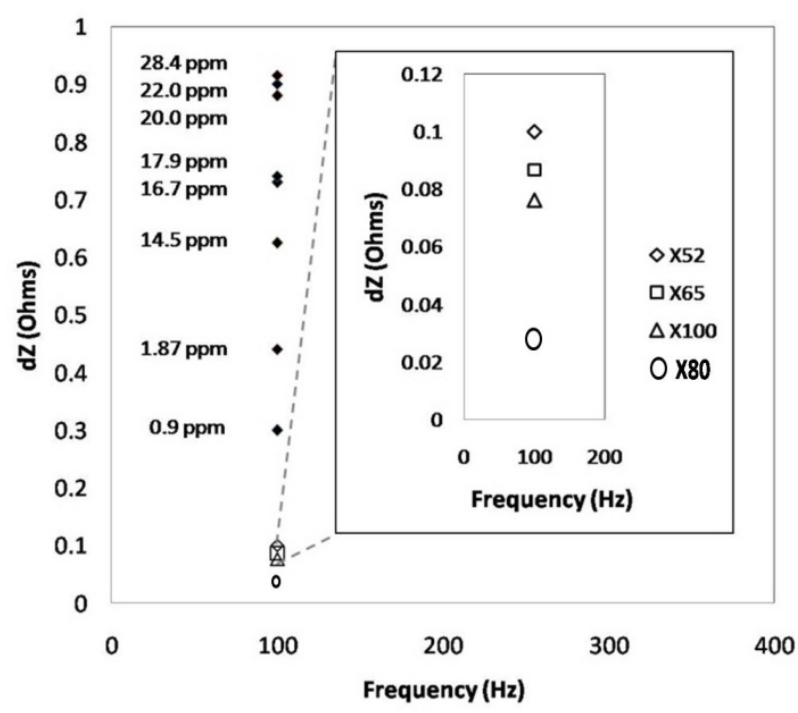

Figure 8. Impedance as a function of hydrogen content for hydrogen charged X80 line pipe steel. Embedded figure is impedance data for a range of uncharged steels with full spectrum of microstructures. Hydrogen content in steel presents a measurable signal [20].

\section{Elastic Wave Measurements}

The measurements of elastic wave velocity, attenuation, nonlinear analyses including frequency scans and fast Fourier data transformation can offer insight about perturbations to the lattice relating to phases, defects, impurities and strain [11,21]. The use of ultrasonic wave velocity measurements to determine the amount of a microstructural constituent is illustrated in Fig. 9.

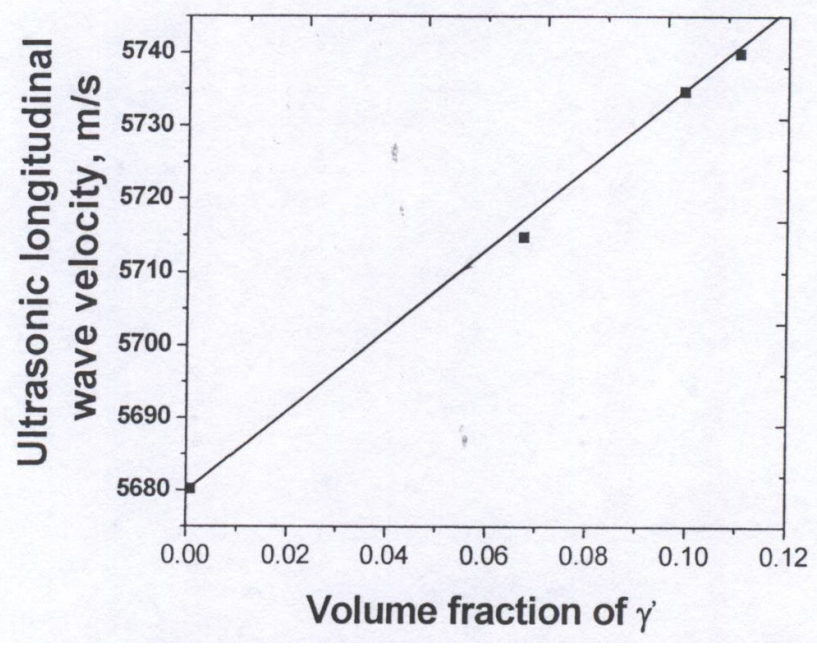

Figure 9. Volume fraction of gamma prime determined by measuring ultrasonic wave velocity [21].

\section{Outlook}

With the use of the proper combination of independent NDE measurements, it is possible to accurately evaluate the microstructure and properties of materials. Different electronic, magnetic, and elastic measurement combinations offer complimentary insights into material properties, and practices for their selection need to be developed for the appropriate applications. This advanced integration of physical property measurements will result in a NDE-based metallography laboratory allowing for non-contact assessment of microstructure, and thus properties, of structural materials. Fig. 10 illustrates the use of multiple advanced NDE measurements to assess microstructures based for nondestructive in-situ elastic-electronic measurements of structural materials in service.

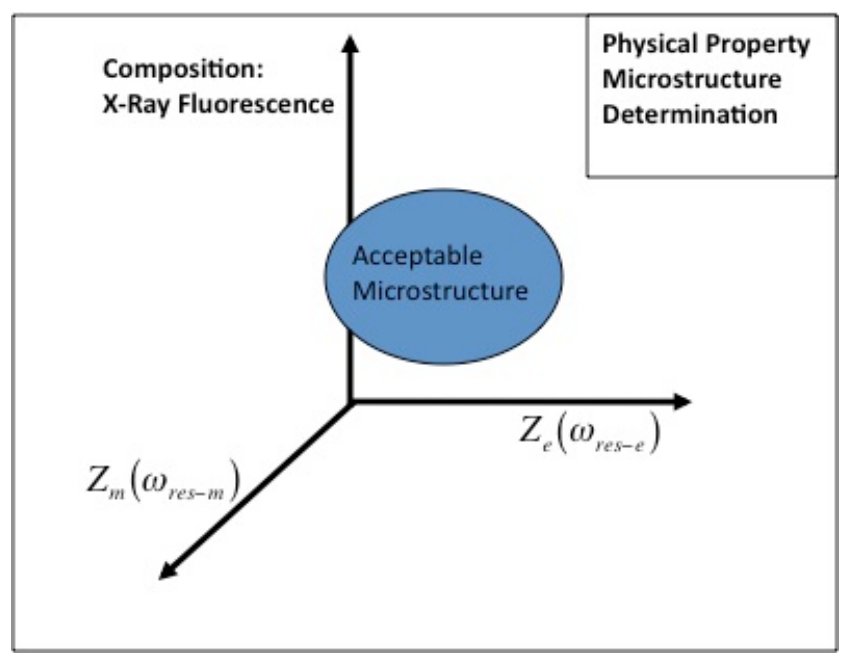

Figure 10. Schematic showing how coupled nondestructive techniques could be used to determine an acceptable microstructure. Using Elastic Impedance, Electronic Impedance (see Table 1), and X-Ray Fluorescence. 


\section{REFERENCES}

[1] K. N. Tu: Ann. Rev. Mater. Sci., Vol. 15 (1985), 147-176.

[2] H. Gleiter: Are There Ways to Synthesize Materials Beyond the Limits of Today?, Met. and Mat.Trans. 40A (7) (2009), 1499-1509.

[3] J. A. Roubidoux, J. E. Jackson, A. N. Lasseigne, B. Mishra and D. L. Olson: Non-linear Analytical Modeling of Interfacial Phenomenon and Nano-size Microstructural Features to Better Correlate NDE Electronic Property Measurements to the Material State, 36th Annual Review of Progress in Quantitative Nondestructive Evaluation, (2010), in press.

[4] D. Zander, I. Maroef, D. L. Olson, and D. Eliezer: Positive Effects of Hydrogen on the Plasticity of $21 / 4 \mathrm{Cr}-1 \mathrm{Mo}$ Steel, Journal of Alloys and Compounds, 356-357 (2003), 809-812.

[5] L.R. Woodyatt, C.T. Sims, and H.M. Beltram: Prediction of Sigma-Type Phase Occurrence from Compositions in Austenitic Superalloys, Transactions of the Metallurgical Society of AIME, 235 (4) (1966), 519-527.

[6] G. Dobmann, N.Meyendorf and E.Schneider: Nondestructive characterization of materials A growing demand for describing damage and service-life-relevant aging processes in plant components, Nucl.Eng. Design, 171 (1-3) (1997), 95-112.

[7] K.V. Rajkumar, S. Vaidyanathan, A. Kumar, T. Jayakumar, B. Raj, K.K. Ray: Characterization of Aging-induced Microstructural Changes in M250 Maraging Steel Using Magnetic Parameters, J. Mag. \& Mag. Mat., 312 (2007), 359-365.

[8] K. V. Rajkumar, A. Kumar, T. Jayakumar, B. Raj, and K. K. Ray: Characterization of Aging Behavior in M250 Grade Maraging Steel Using Ultrasonic Measurements, Met. Mat. Trans. 38A (2) (2007), 236-243.

[9] M. N. Rao: Progress in Understanding the Metallurgy of 18\% Nickel Maraging Steels, Int. J. Mater. Res., 97 (11) (2006), 1594-1607.

[10] Y. Snir, M. Pinkas, Y. Gelbstein, O. Yeheskel and A. Landau: Applying TEP Measurements to Assess the Aging Stage of Maraging 250 Steel, 34th Annual Review of Progress in Quantitative Nondestructive Evaluation, 975 (2006), 1148-1153.

[11] J. E. Jackson, A. N. Lasseigne-Jackson, Y. D. Park, B. Mishra, and D. L. Olson: The Future of Microstructural Characterization: An Electron Metallography Laboratory, 34th Annual Review of Progress in Quantitative Nondestructive Evaluation, 975 (2008), 1085-1092.
[12] F. G. Caballero, A. García-Junceda, C. Capdevila, C. García de Andre: Precipitation of $M_{23} C_{6}$ Carbides: Thermoelectric Power Measurements, Scripta Mat., 52 (2005), 501-505.

[13] Y. Jae-Kyung, L. Byong-Whi, H.C. Kim: Nondestructive Evaluation of Isothermally Annealed $12 \% \mathrm{CrMoV}$ Steel by Magnetic BN Measurement, J. Mag. \& Mag. Mat., 130 (1994), 81-91.

[14] A. N. Lasseigne and D. L. Olson: Development of Non Destructive Techniques for Characterization of Residual Stresses in Advanced Materials, Materials Evaluation, 66 (10) (2008), 1077-1083.

[15] C. A. VanHorn, D. L. Olson, and B. Mishra: Assessing Residual Stress on Machined Uranium Using Nondestructive Thermoelectric Power Coefficient and Induced Impedance Measurements," 35th Annual Review of Progress in Quantitative Nondestructive Evaluation, 1096 (2009), 1317-1324.

[16] A. N. Lasseigne-Jackson, J. M Anton and T.A. Siewert, D.L. Olson, B. Mishra and J.E. Jackson: Advanced Nondestructive Measurement Schemes to Actively Monitor Hydrogen Content in Steel Pipeline, 34th Annual Review of Progress in Quantitative Nondestructive Evaluation, 975 (2008), 1117-1124.

[17] A.N. Lasseigne-Jackson, J. Anton, J.E. Jackson, D.L. Olson, and B. Mishra: Development of Electromagnetic Techniques for Hydrogen Content Assessment in Coated Linepipe, 33rd Annual Review of Progress in Quantitative Nondestructive Evaluation, 894 (2007), 1159-1166.

[18] A.N. Lasseigne, K. Koenig, D.L. Olson, J.E. Jackson and B. Mishra: Real-Time Low Frequency Impedance Measurements for Determination of Hydrogen content in Pipeline Steel, 35th Annual Review of Progress in Quantitative Nondestructive Evaluation, 1096 (2009), 1283-1290.

[19] K. Koenig, A. N. Lasseigne, J. W. Cisler, B. Mishra, and D. L. Olson: Non-Contact Non-Destructive Hydrogen and Microstructural Assessment of Steel Welds, Proc. EPRI Welding Conf. (2009), to be published.

[20] E. A. Pfeif, A. N. Lasseigne, K. Krzywosz, E. V. Mader, B. Mishra, and D. L. Olson: Characterization of Hydrogen Content in Zircaloy 4 Nuclear Fuel Cladding Utilizing NDE Electronic Property Measurements, 36th Annual Review of Progress in Quantitative Nondestructive Evaluation, (2010), to be published.

[21] T. Jayakumar, B. Raj, H. Williams, W. Arnold: Influence of Microstructure on Ultrasonic Velocity in Nimonic Alloy PE16, 17th Annual Review of Progress in Quantitative Nondestructive Evaluation, 10B (1991), 1693-1700. 